\title{
Dissociation of hydrogen molecules on the clean and hydrogen-preadsorbed $\mathrm{Be}(0001)$ surface
}

\author{
Yanfang $\mathrm{Li}^{1,2}$, Yu Yang${ }^{2}$, Bo Sun ${ }^{2}$, Yinghui Wei ${ }^{1}$, Ping Zhang ${ }^{2,3 *}$ \\ ${ }^{1}$ College of Materials Science and Engineering, \\ Taiyuan University of Technology, Taiyuan 030024, People's Republic of China \\ ${ }^{2}$ LCP, Institute of Applied Physics and Computational Mathematics, \\ P.O. Box 8009, Beijing 100088, People's Republic of China and \\ ${ }^{3}$ Center for Applied Physics and Technology, \\ Peking University, Beijing 100871, Peoples Republic of China
}

(Dated: October 29, 2018)

\begin{abstract}
Using first-principles calculations, we systematically study the potential energy surfaces and dissociation processes for hydrogen molecules on the clean and hydrogen-preadsorbed $\operatorname{Be}(0001)$ surfaces. It is found that the most energetically favored dissociation channel for $\mathrm{H}_{2}$ molecules on the clean Be surface is at the surface top site, with the minimum energy barrier of $0.75 \mathrm{eV}$. It is further found that after dissociation, hydrogen atoms do not like to cluster with each other, as well as to penetrate into subsurface sites. For the hydrogen-preadsorbed $\mathrm{Be}(0001)$ surface, the smallest dissociation energy barrier for $\mathrm{H}_{2}$ molecules is found to be $0.50 \mathrm{eV}$, which is smaller than the dissociation energy barrier on a clean Be(0001) surface. The critical dependence of the dissociation energy barriers for $\mathrm{H}_{2}$ molecules on their horizontal distances from the preadsorbed hydrogen atom is revealed. Our studies well describe the adsorption behaviors of hydrogen on the $\operatorname{Be}(0001)$ surface.
\end{abstract}

PACS numbers: 68.43.Bc, 68.43.Fg, 68.43.Jk, 73.20.Hb

\footnotetext{
* Corresponding author. E-mail address: zhang_ping@iapcm.ac.cn
} 


\section{INTRODUCTION}

The dissociative adsorption of hydrogen molecules on metal surfaces is of great importance since it is a crucial step in many application processes, such as hydrogen storage for fuels ${ }^{1}$, hydrogen caused embrittlement ${ }^{2}$, and heterogeneous catalysis ${ }^{3} \underline{4}$. Among all the metals, simple $s p$-metals (metals with only $s p$ valence electrons), such as Be and $\mathrm{Mg}$, are good candidates for hydrogen storage materials, and have vast applications in industries where hydrogen caused embrittlement need to be prevented. So the adsorption and dissociation of hydrogen molecules on these $s p$-metals need special concerns. For the $\mathrm{H}_{2} / \mathrm{Mg}(0001)$ system, many studies have already been carried out ${ }^{5-7}$. It is found that the most energetically favorable site for the dissociation of molecule $\mathrm{H}_{2}$ on the $\mathrm{Mg}(0001)$ surface is the bridge site ${ }^{5.7}$, and the theoretically calculated energy barrier for dissociation is in good accordance with the experimentally observed values ${ }^{7}$. Similar to the $\operatorname{Mg}(0001)$ surface, the $\operatorname{Be}(0001)$ surface also has considerable $s$ and $p$ electronic states distributing around the Fermi energy ${ }^{8}$, which makes it a good prototype to study the adsorption behaviors of $\mathrm{H}_{2}$ on $s p$-metals. However, theoretical studies have not been carried out on the dissociation process of $\mathrm{H}_{2}$ molecules on the $\mathrm{Be}(0001)$ surface yet.

On the other hand, Be has vast applications in modern nuclear devices. In the international thermonuclear experimental reactor (ITER), Be contributes the major part of the first wall ${ }^{\underline{9}}$, based on its low atomic number, ability to remove oxygen from the plasma, and ability to adsorb residue gases composed of light atoms as $\mathrm{C}, \mathrm{H}$ and $\mathrm{O}^{10} \underline{-12}$. For these important applications, the adsorption of small molecules such as $\mathrm{H}_{2}, \mathrm{O}_{2}$ and $\mathrm{H}_{2} \mathrm{O}$ on the Be surfaces need to be specially investigated. Actually, the molecular adsorption and dissociation of $\mathrm{H}_{2} \mathrm{O}^{13,14}$ and $\mathrm{O}_{2} \underline{\underline{15}-17}$ have already been studied systematically. Therefore, studying the adsorption of hydrogen on the $\mathrm{Be}(0001)$ surface not only gives us knowledge about the common characters of interactions between $\mathrm{H}_{2}$ and $s p$-metals, but also provides us information on how to advance the usages of Be materials in nuclear reactors.

Correspondingly, the adsorption of hydrogen atoms on the $\mathrm{Be}(0001)$ surface has been widely studied, including the adsorption of different coverage $\underline{\underline{18}-20}$. It is found that at low coverage, $\mathrm{H}$ atoms choose to adsorb at surface hcp hollow sites, while at the 1 monolayer (ML) coverage, the adsorption at surface bridge sites is energetically more favorable ${ }^{19}$. However, as the first step towards surface hydrogenation, the adsorption and dissociation of molecular 
$\mathrm{H}_{2}$ on the $\mathrm{Be}(0001)$ surface is more important for the above research backgrounds. And to our surprise, in contrary to the vast studies on the adsorption of atomic $\mathrm{H}$, the adsorption and dissociation of molecular $\mathrm{H}_{2}$ on the $\mathrm{Be}(0001)$ surface has not been studied at all. Based on these backgrounds, here we perform first-principles calculations to systematically study the adsorption and dissociation of molecular $\mathrm{H}_{2}$, the distribution of dissociated $\mathrm{H}$ atoms, and the adsorption and dissociation of molecular $\mathrm{H}_{2}$ on the hydrogen-preadsorbed $\mathrm{Be}(0001)$ surfaces. The rest of this paper is organized as follows. In Sec. II, we give details of the firstprinciples total energy calculations, which is followed in Sec. III by our results for the PESs of molecular $\mathrm{H}_{2}$ on the clean $\mathrm{Be}(0001)$ surface, where the energetically favored dissociation paths and the dissociation energy barriers are presented and discussed. In Sec. IV, we study the diffusion and penetration of hydrogen atoms on the Be(0001) surface, and discuss the distribution properties of the dissociated hydrogen atoms. Then the PESs for molecular $\mathrm{H}_{2}$ on hydrogen-preadsorbed Be(0001) surface are calculated and discussed in Sec. V, similar to what we did in Sec. III. And finally in Sec. VI, we give our conclusions.

\section{METHODS}

Our calculations were performed within density functional theory (DFT) using the Vienna $a b$-initio simulation package (VASP) $\underline{\underline{21}}$. The PW91 $\frac{22}{\underline{22}}$ generalized gradient approximation and the projector-augmented wave potential $\underline{23}$ were employed to describe the exchangecorrelation energy and the electron-ion interaction, respectively. The cutoff energy for the plane wave expansion was set to $300 \mathrm{eV}$. The $\mathrm{Be}(0001)$ surface was modeled by a slab composing of five atomic layers and a vacuum region of $20 \AA$. The $2 \times 2$ and $3 \times 3$ supercell (in which each monolayer contains four and nine Be atoms) were adopted respectively in the study of the $\mathrm{H}_{2}$ adsorption on the clean and hydrogen-preadsorbed $\mathrm{Be}(0001)$ surfaces. Our test calculations have shown that the $2 \times 2$ supercell is sufficiently large to avoid the interaction between adjacent hydrogen molecules. And the $3 \times 3$ supercell was used to explore more carefully the effect of the preadsorbed $\mathrm{H}$ atom. Integration over the Brillouin zone was done using the Monkhorst-Pack scheme ${ }^{24}$ with $11 \times 11 \times 1$ and $7 \times 7 \times 1$ grid points respectively for the $2 \times 2$ and $3 \times 3$ supercell. A Fermi broadening 25 of $0.1 \mathrm{eV}$ was chosen to smear the occupation of the bands around the Fermi energy $\left(\mathrm{E}_{F}\right)$ by a finite- $T$ Fermi function and extrapolating to $T=0 \mathrm{~K}$. The calculation of the potential energy surface for 
molecular $\mathrm{H}_{2}$ was interpolated to 209 points with different bond length $\left(d_{\mathrm{H}-\mathrm{H}}\right)$ and height $\left(h_{\mathrm{H}_{2}}\right)$ of $\mathrm{H}_{2}$ at each surface site. The calculated lattice constant of bulk $\mathrm{Be}(a, c)$ and the bond length of a free $\mathrm{H}_{2}$ molecule are $2.26 \AA, 3.56 \AA$ and $0.75 \AA$, respectively, in good agreement with the experimental values of $2.285 \AA, 3.585 \AA^{26}$ and $0.74 \AA \underline{27}$.

\section{ADSORPTION AND DISSOCIATION OF MOLECULAR $\mathrm{H}_{2}$ ON THE CLEAN BE(0001) SURFACE}

The geometry and electronic properties of the clean $\mathrm{Be}(0001)$ surface is first investigated. In comparison with that in bulk Be, the electronic density of states (DOS) at the Fermi energy $\left(\mathrm{E}_{F}\right)$ has a relatively small value, the DOS at $\mathrm{E}_{F}$ in the clean $\operatorname{Be}(0001)$ surface is prominently enhanced ${ }^{17}$. From wavefunction analysis, it is found that the electronic states around $\mathrm{E}_{F}$ are mainly Be $2 p$ states, and mainly accumulate within the two topmost Be layers. Due to this pronounced surface charge redistribution, the two outmost $\operatorname{Be}(0001)$ layers relax significantly from the bulk values. The first-second interlayer contraction is $3.8 \%$ and the second-third interlayer expansion is nearly $1.2 \%$, which is in agreement with recent first-principles calculations ${ }^{28}$ and comparable with experimental measurements ${ }^{29}$.

After geometry optimization for the Be(0001) surface, we build our model to study the two-dimensional (2D) PES cuts for $\mathrm{H}_{2}$ on the relaxed Be surface. As shown in Fig. 1, there are four different high-symmetry sites on the Be (0001) surface, respectively the top, bridge (bri), hcp and fcc hollow sites. At each surface site, an adsorbed $\mathrm{H}_{2}$ has three different high-symmetry orientations, respectively along the $x$ (i.e., [11 $\overline{2} 0]$ ), $y$ (i.e., [1100]), and $z$ (i.e., [0001]) directions. Herein, we use top- $x, y, z$, bri- $x, y, z$, hcp- $x, y, z$ and fcc- $x, y, z$ respectively to represent the twelve high-symmetry channels for the adsorption of $\mathrm{H}_{2}$ on the Be surface. We have also tested several low-symmetry adsorption channels, and found that the dissociation energy barriers for $\mathrm{H}_{2}$ along these low-symmetry channels are always larger than that along high-symmetry channels. Similar results have also been obtained for the $\mathrm{O}_{2} / \mathrm{Pb}(111)$ system where molecular adsorption of $\mathrm{O}_{2}$ only occurs at surface high-symmetry $\operatorname{sites}^{30}$. Therefore, we will only discuss the PES cuts along the high-symmetry channels.

From our PES calculations, we find that there are no molecular adsorption states for $\mathrm{H}_{2}$ on the $\mathrm{Be}(0001)$ surface. This finding is similar to the adsorption of $\mathrm{H}_{2}$ on the $\mathrm{Mg}(0001)$ surface $^{5,7}$. The calculated 2D PES cuts along the top- $x, y$ and $z$ channels are respectively 


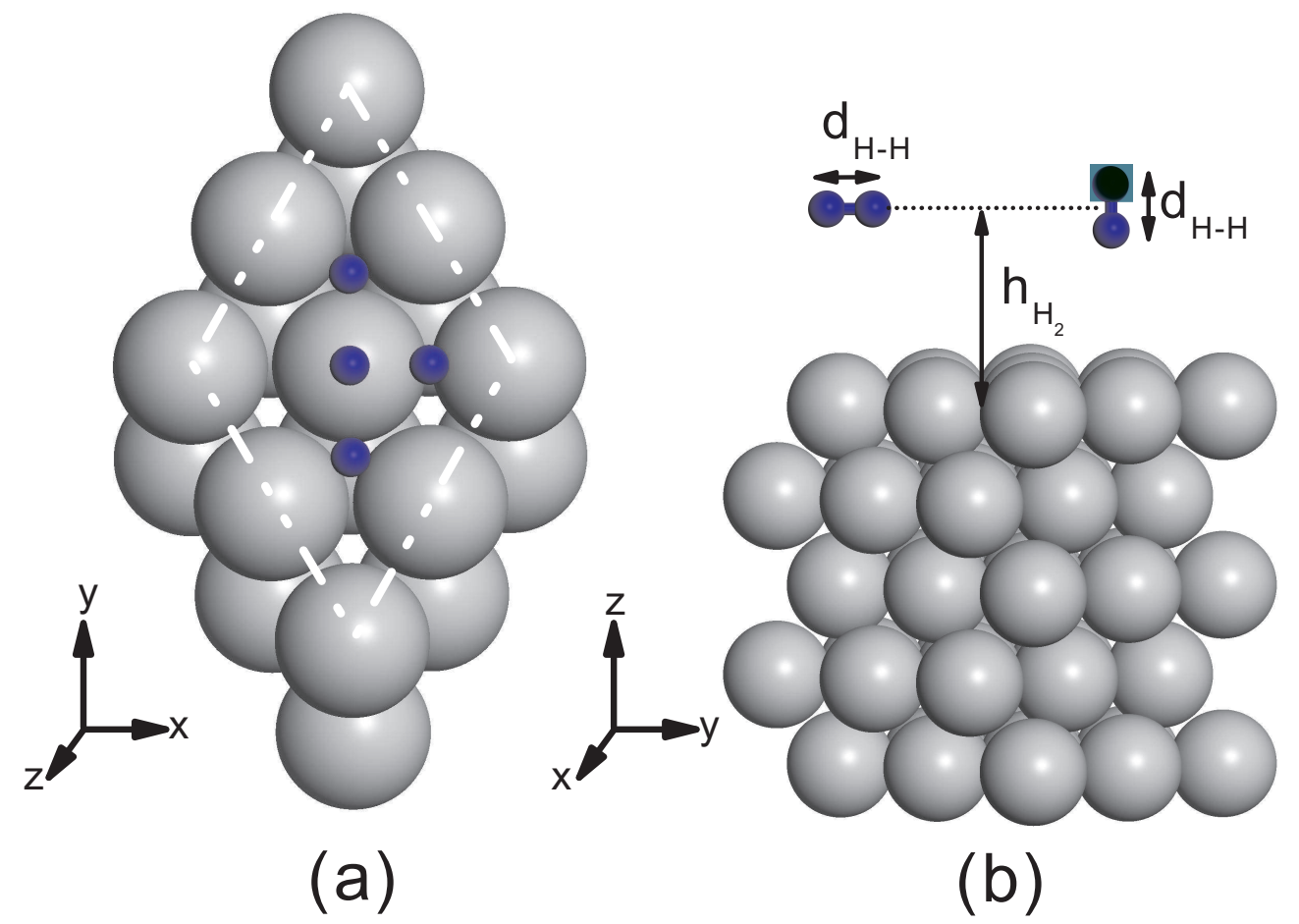

FIG. 1: (Color online). (a) The $p(2 \times 2)$ surface cell of Be(0001) and four on-surface adsorption sites. Here only the outmost two layers of the surface are shown. (b) The sketch map showing that the molecule (with vertical or parallel orientation) is initially away from the surface with a hight $h_{\mathrm{H}_{2}}$.

listed in Figs. 2(a), (b) and (c). The other three PES cuts along the bri-, hcp- and fcc- $z$ channels have similar energy distributions with the top- $z$ channel, and the energies needed to separate the two $\mathrm{H}$ atoms to $2.0 \AA$ are all about $2.08 \mathrm{eV}$ for these four channels. The top- $x$ and $y$ channels are two nearly degenerate channels, as we can see from Fig. 2(d) that the minimum energy path along these two channels are the same with different heights. These two channels also have the lowest energy barriers for the dissociation of $\mathrm{H}_{2}$ among the twelve adsorption channels. Herein, the dissociation of $\mathrm{H}_{2}$ molecules on the clean $\mathrm{Be}(0001)$ surface is different from on the clean $\mathrm{Mg}(0001)$ surface, where the bri- $y$ channel is the most energetically favored dissociation channe $\mathrm{I}^{5,7}$. Besides, it is interesting to find from Fig. 2(d) that the minimum energy paths along the top- $x(y)$ and $z$ channels have a cross point at the molecular height of $1.64 \AA$, which means that the $\mathrm{H}_{2}$ molecule would firstly orients perpendicular, and then rotates to be parallel to the Be surface during its dissociation process. 

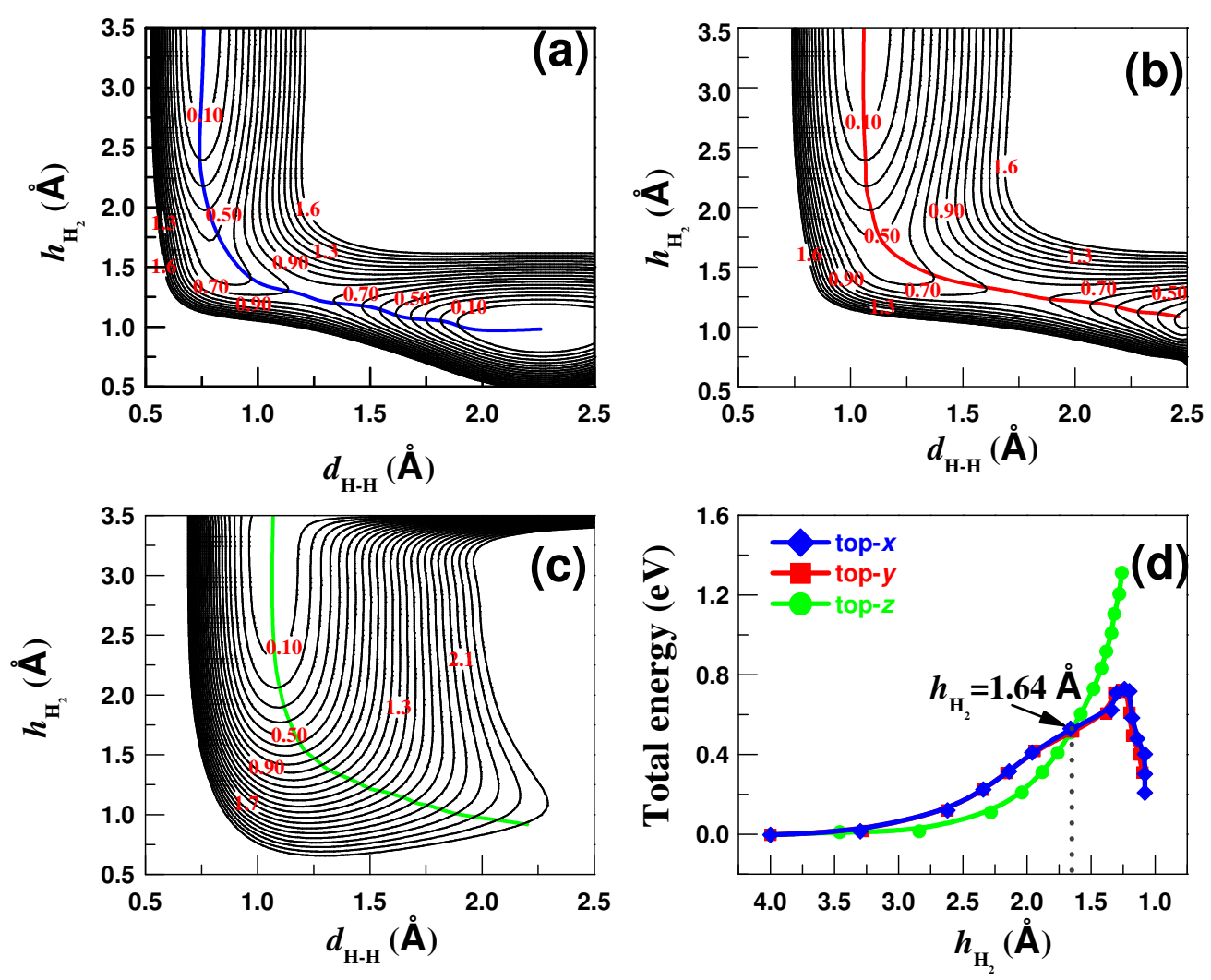

FIG. 2: (Color online). Contour plots of the two-dimensional cuts of the potential energy surfaces (PESs) for the $\mathrm{H}_{2} / \mathrm{Be}(0001)$ system as a function of the bond length $\left(d_{\mathrm{H}-\mathrm{H}}\right)$ and molecular height of $\mathrm{H}_{2}\left(h_{\mathrm{H}_{2}}\right)$, along the top- $x(\mathrm{a}), y(\mathrm{~b})$ and $z$ (c) channels. (d) The corresponding minimum energy paths for the dissociative adsorption of molecular $\mathrm{H}_{2}$ along the top- $x, y, z$ channels. In all the figures, the total energy of an isolated $\mathrm{H}_{2}$ molecule plus a clean $\mathrm{Be}(0001)$ surface is set to zero.

The minimum energy path along the top-, bri-, hcp- and fcc- $x, y$ channels are drawn together in Fig. 3. The corresponding dissociation energy barrier $(\Delta \mathrm{E})$ along these channels are summarized in Table I. Besides of the top- $x, y$ channels, along which $\mathrm{H}_{2}$ molecules can easily dissociate at room temperatures in front of the $0.75 \mathrm{eV}$ energy barrier, the bri- $y$ channel is also a probable dissociation path since its energy barrier is only $0.16 \mathrm{eV}$ higher than the top- $x(y)$ channel. Remarkably, the lowest energy barrier for dissociation of $\mathrm{H}_{2}$ molecules is much smaller on the $\mathrm{Be}(0001)$ surface than on the $\mathrm{Mg}(0001)$ surface $\mathrm{5}^{\underline{7}}$. This is probably a good news for hydrogen storage researchers. Form Fig. 3 and Table I, one may also note that the dissociation energy barriers for $\mathrm{H}_{2}$ is always larger at surface hcp hollow sites than fcc hollow sites. Considering that there are more electrons distributing at the surface hcp hollow site ${ }^{31}$, this finding reflects that charge transfer from the Be surface 




FIG. 3: (Color online). The minimum energy paths for the dissociative adsorption of molecular $\mathrm{H}_{2}$ along all the considered adsorption channels with high symmetries. The total energy of an isolated $\mathrm{H}_{2}$ molecule plus a clean $\mathrm{Be}(0001)$ surface is set to zero.

to $\mathrm{H}_{2}$ is important for the dissociation of $\mathrm{H}_{2}$. The bond length $\left(d_{\mathrm{H}-\mathrm{H}}(\mathrm{TS})\right)$ and molecular height of $\mathrm{H}_{2}\left(h_{\mathrm{H}_{2}}\right.$ (TS)) at the corresponding transition states are also included in Table I. Although the interactions between $\mathrm{H}$ atoms and the $\mathrm{Be}(0001)$ surface have long been studied, our results concerning the dissociation energy barrier and minimum energy paths are still meaningful.

IV. DIFFUSION, PENETRATION AND DISTRIBUTION OF DISSOCIATED HYDROGEN ATOMS

The PES cut for one $\mathrm{H}$ atom on the $\mathrm{Be}(0001)$ surface is also calculated to study its diffusion characters. Our calculated PES is shown in Fig. 4(a), with the energy path crossing the four high-symmetry surface sites shown in Fig. 4(b). One can see that the hcp 
TABLE I: The dissociation energy barriers for $H_{2}$ along the high symmetry adsorption channels, and the corresponding bond length $\left(d_{\mathrm{H}-\mathrm{H}}(\mathrm{TS})\right)$ and molecular height of $\mathrm{H}_{2}\left(h_{\mathrm{H}_{2}}(\mathrm{TS})\right)$ at the transition states.

\begin{tabular}{cccc}
\hline adsorption channel & $\Delta \mathrm{E}(\mathrm{eV})$ & $d_{\mathrm{H}-\mathrm{H}}(\mathrm{TS})(\AA)$ & $h_{\mathrm{H}_{2}}(\mathrm{TS})(\AA)$ \\
\hline top- $x$ & 0.75 & 1.24 & 1.26 \\
top-y & 0.75 & 1.74 & 1.26 \\
bri- $x$ & 1.62 & 1.24 & 1.40 \\
bri-y & 0.91 & 0.88 & 1.40 \\
hcp- $x$ & 1.28 & 1.04 & 1.28 \\
hcp- $y$ & 1.29 & 1.04 & 1.32 \\
fcc- $x$ & 1.18 & 1.06 & 1.26 \\
fcc- $y$ & 1.22 & 1.06 & 1.26 \\
\hline
\end{tabular}

and fcc hollow sites are two minimum energy locations for the $\mathrm{H}$ atom, while the bridge site is the saddle point in the diffusion pathway, and the top sites are maxima of the PES. As shown in Fig. 4(b) the energy barrier for one $\mathrm{H}$ atom to diffuse from the hcp (fcc) to fcc (hcp) hollow site is $0.35(0.28) \mathrm{eV}$, which is very small and can be easily overcome at room temperatures. So after dissociation, the $\mathrm{H}$ atoms will easily diffuse around on the $\mathrm{Be}(0001)$ surface.

As has been pointed out, the adsorption of $\mathrm{H}$ atoms energetically prefers the surface hollow sites at low coverage on the $\mathrm{Be}(0001)$ surface $^{19}$. We then study the penetration of $\mathrm{H}$ atoms from these surface hcp and fcc hollow sites to the subsurface sites. There are three different kinds of high-symmetry subsurface sites. The octahedral site (octa) lies just underneath the on-surface fcc site, and one tetrahedral site (tetra) lies below the on-surface hcp site. A second tetrahedral site (tetra*) is located directly below a first-layer metal atom. However, the direct hydrogen penetration into the tetra* site from the on-surface adsorption without bypassing the other subsurface sites is very unfavorable, since this site is located beneath a surface Be atom. So the penetration into the tetra* site will not be discussed. And our calculated results for penetrations into the other two subsurface sites are shown in Figs. 5(a) and (b) respectively. It is clearly shown in Fig. 5(a) that there is no stable adsorption states for atomic $\mathrm{H}$ in the subsurface tetra site, and the energy barrier for one $\mathrm{H}$ 

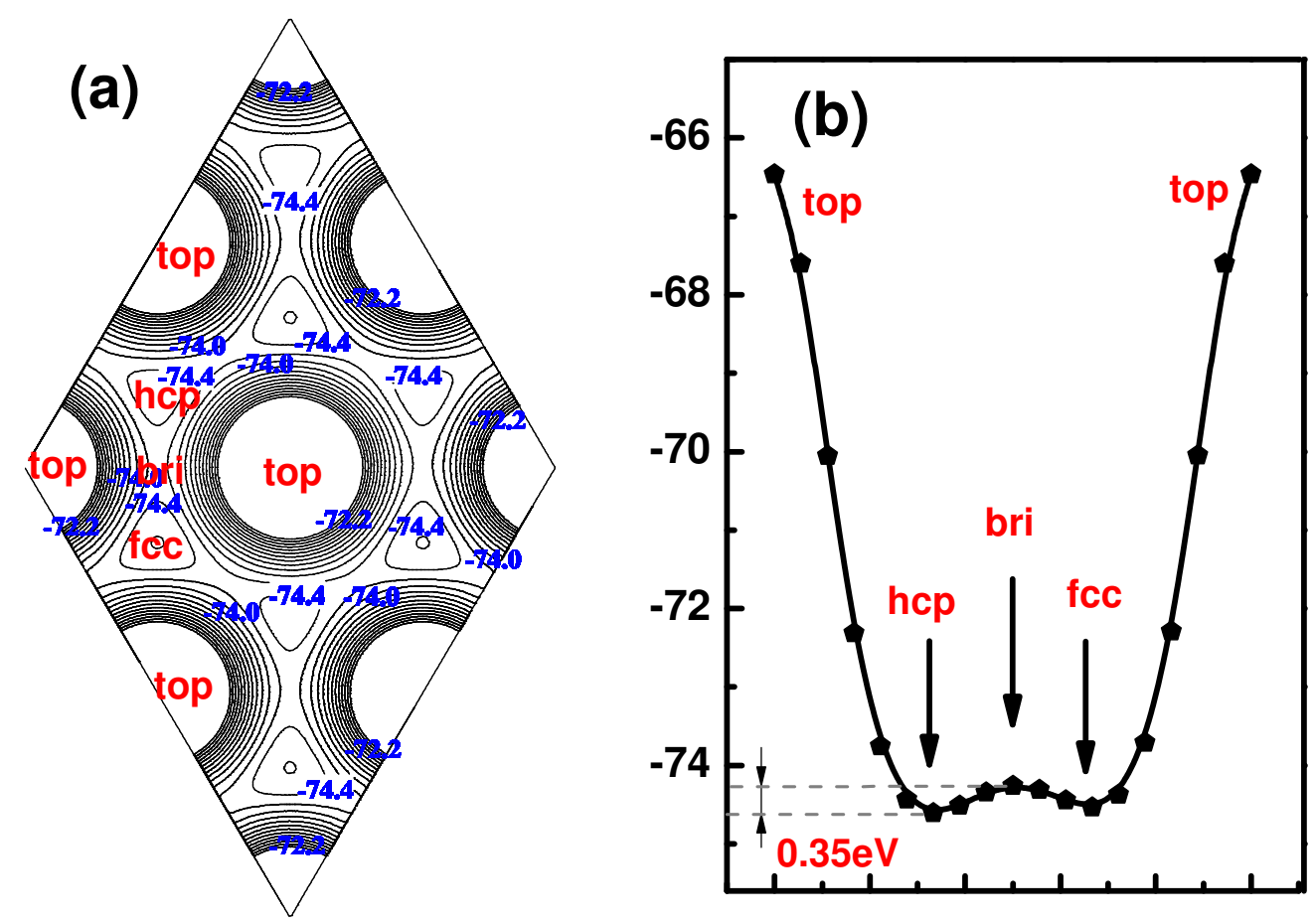

FIG. 4: (Color online). (a) Contour plots of the potential energy surface distribution for one $\mathrm{H}$ atom on the $\mathrm{Be}(0001)$ surface, with the height of $h_{\mathrm{H}_{2}}=0.85 \AA$. (b) The energy curve for one $\mathrm{H}$ atom crossing the four high-symmetry surface sites on the Be(0001) surface.

atom to penetrate through the Be surface is larger than $1.5 \mathrm{eV}$. As shown in Fig. 5(b), for subsurface adsorption, the $\mathrm{H}$ atom will stay at the octa site. However, the energy barrier from on-surface fcc hollow site to subsurface octa site is as large as $1.25 \mathrm{eV}$. In addition, the subsurface adsorption state is much less stable than the on-surface adsorption state at the fcc hollow site, and the energy barrier for the $\mathrm{H}$ atom to penetrate from subsurface back to on-surface site is very small $(\sim 0.07 \mathrm{eV})$. So after dissociation, the $\mathrm{H}$ atoms have a much larger probability to stay on the clean Be(0001) surface than penetrating into the subsurface sites.

After the systematic studies on the diffusion and penetration energy barriers, we then analyze the distribution properties for the dissociated $\mathrm{H}$ atoms. Since the penetration of atomic $\mathrm{H}$ is found to be very hard, and the subsurface adsorption is always less stable than on-surface adsorption, we will mainly discuss the distribution for on-surface adsorption of $\mathrm{H}$ atoms. And we focus on the total energy calculation for the adsorption system of dissociated $\mathrm{H}$ atoms at different hollow sites. Figures 6(a), (b) and (c) respectively shows three different 


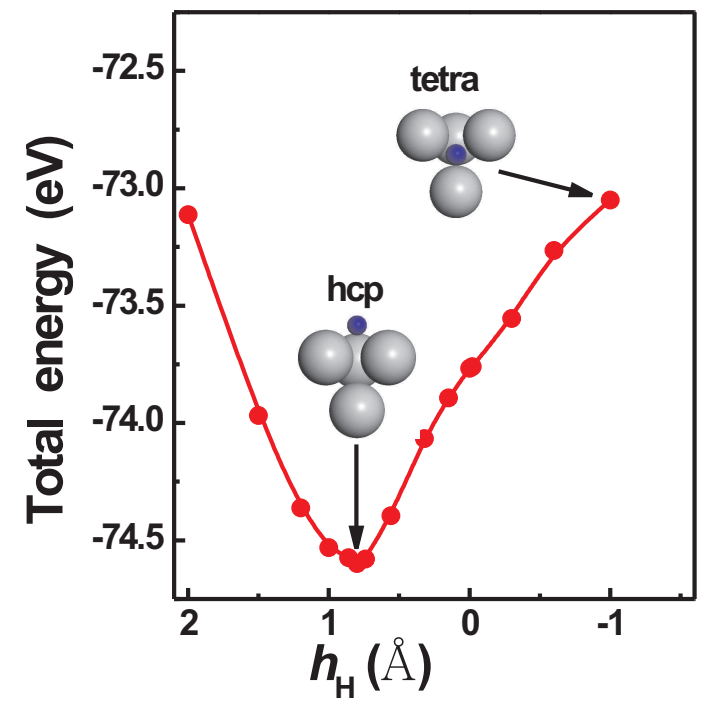

(a)

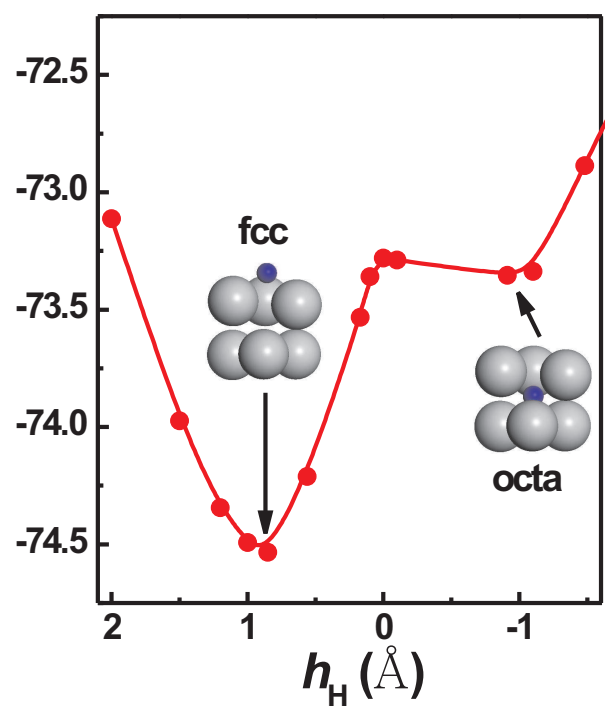

(b)

FIG. 5: (Color online). The energy curve for one $\mathrm{H}$ atom penetrating from the surface hcp to subsurface tetra sites (a), and from the surface fcc to subsurface octa sites (b).

adsorption structures after geometry optimizations, in which the two dissociated $\mathrm{H}$ atoms respectively adsorb at two adjacent fcc and hcp hollow sites, two separate fcc hollow sites and two separate hcp hollow sites. Obviously, the H atoms are more clustered to each other in Fig. 6(a) than in Figs. 6(b) and (c). And the structural difference between Figs. 6(b) and (c) lies in that the $\mathrm{H}$ atoms residue at fcc and hcp hollow sites respectively. Our calculated total energies are respectively $-77.70,-77.98$ and $-78.07 \mathrm{eV}$ for the three distributions in Figs. 6(a), (b) and (c). It means that the adsorption state of two $\mathrm{H}$ atoms always has a higher total energy when they are clustered. So after dissociation, the $\mathrm{H}$ atoms will not cluster with each other, instead, they will distribute uniformly on the clean Be(0001) surface. In addition, the adsorption of $\mathrm{H}$ atoms at surface hcp hollow sites are always stabler than at surface fcc hollow sites.

\section{ADSORPTION OF $\mathrm{H}_{2}$ ON HYDROGEN-PREADSORBED BE(0001) SUR- FACE}

From the previous results, we see that $\mathrm{H}_{2}$ molecules easily dissociate on the $\mathrm{Be}(0001)$ surface, and the dissociated $\mathrm{H}$ atoms tends to distribute uniformly on the surface. Herein, we further study the adsorption of $\mathrm{H}_{2}$ molecules on the hydrogen-preadsorbed $\mathrm{Be}(0001)$ surface. 


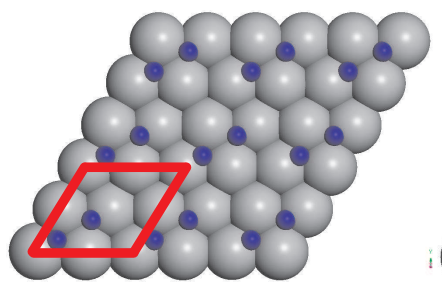

(a)

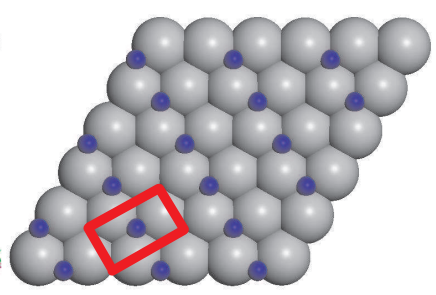

(b)

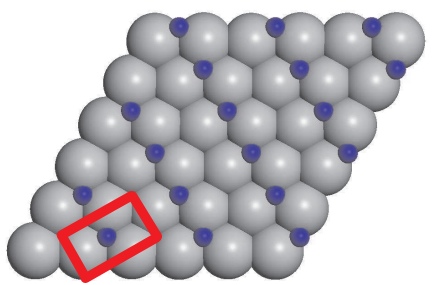

(c)

FIG. 6: (Color online). Three kinds of configurations for atomic $\mathrm{H}$ adsorbates at the same coverage of 0.5 ML. The H adatoms in (a) is arranged to be clustered, while in (b) and (c) have uniform distributions. The calculated total energies show that the adsorption in (c) is the most stable.

The preadsorbed $\mathrm{H}$ atom is set at a surface hcp hollow site, since it is the most energetically favored adsorption site for $\mathrm{H}$ atoms at low coverage ${ }^{19}$. And in order to compare the adsorption of $\mathrm{H}_{2}$ molecules near to and away from the preadsorbed $\mathrm{H}$ atom, a $3 \times 3$ supercell with 9 Be atoms at each monolayer is adopted, with the k-points grid for integrations over the Brillouin zone changed to be $7 \times 7 \times 1$. Our structural model for the hydrogen-preadsorbed $\mathrm{Be}(0001)$ surface is shown in Fig. 7, with the considered high symmetry sites respectively denoted as $\mathrm{T} 1 \sim \mathrm{T} 5, \mathrm{~B} 1 \sim \mathrm{B} 3, \mathrm{~F} 1 \sim \mathrm{F} 2$ and $\mathrm{H} 1 \sim \mathrm{H} 2$. Since a $\mathrm{H}_{2}$ molecule has different possible orientations while adsorbing at each high symmetry sites, we here use for example, T1- $x$ to represent for the adsorbing channel of a $\mathrm{H}_{2}$ molecule orienting along the $x$ (i.e., [11 $\left.\overline{2} 0\right]$ ) direction at the $\mathrm{T} 1$ site.

Among all the calculations, we find no molecular adsorption states of $\mathrm{H}_{2}$ on the hydrogenpreadsorbed Be(0001) surface. The calculated 2D-PES cuts for the H1-x, T2-y, B3-y and T3- $x$ channels are respectively shown in Figs. 8(a), (b), (c) and (d). As shown in Fig. 8(a), the dissociation energy barrier for a $\mathrm{H}_{2}$ molecule directly on the precovered $\mathrm{H}$ atom is 3.61 $\mathrm{eV}$. Since the dissociation energy barrier for a $\mathrm{H}_{2}$ molecule at the hcp hollow site of a clean $\mathrm{Be}(0001)$ surface is only $1.28 \mathrm{eV}$ as been listed in Table I, the preadsorbed $\mathrm{H}$ atom actually makes the dissociation of $\mathrm{H}_{2}$ molecule on top of it much harder. Moreover, as shown in Fig. 8(b), the dissociation energy barrier for a $\mathrm{H}_{2}$ molecule around the preadsorbed $\mathrm{H}$ atom along the $\mathrm{T} 2-y$ channel is $1.70 \mathrm{eV}$. In comparison with the energy barrier of $0.75 \mathrm{eV}$ for a $\mathrm{H}_{2}$ molecule at the top site of a clean $\mathrm{Be}(0001)$ surface, the dissociation of a $\mathrm{H}_{2}$ molecule becomes harder too around a preadsorbed $\mathrm{H}$ atom. However, when the $\mathrm{H}_{2}$ molecule is enough far away from the preadsorbed H atom, the dissociation energy barrier can be lower 


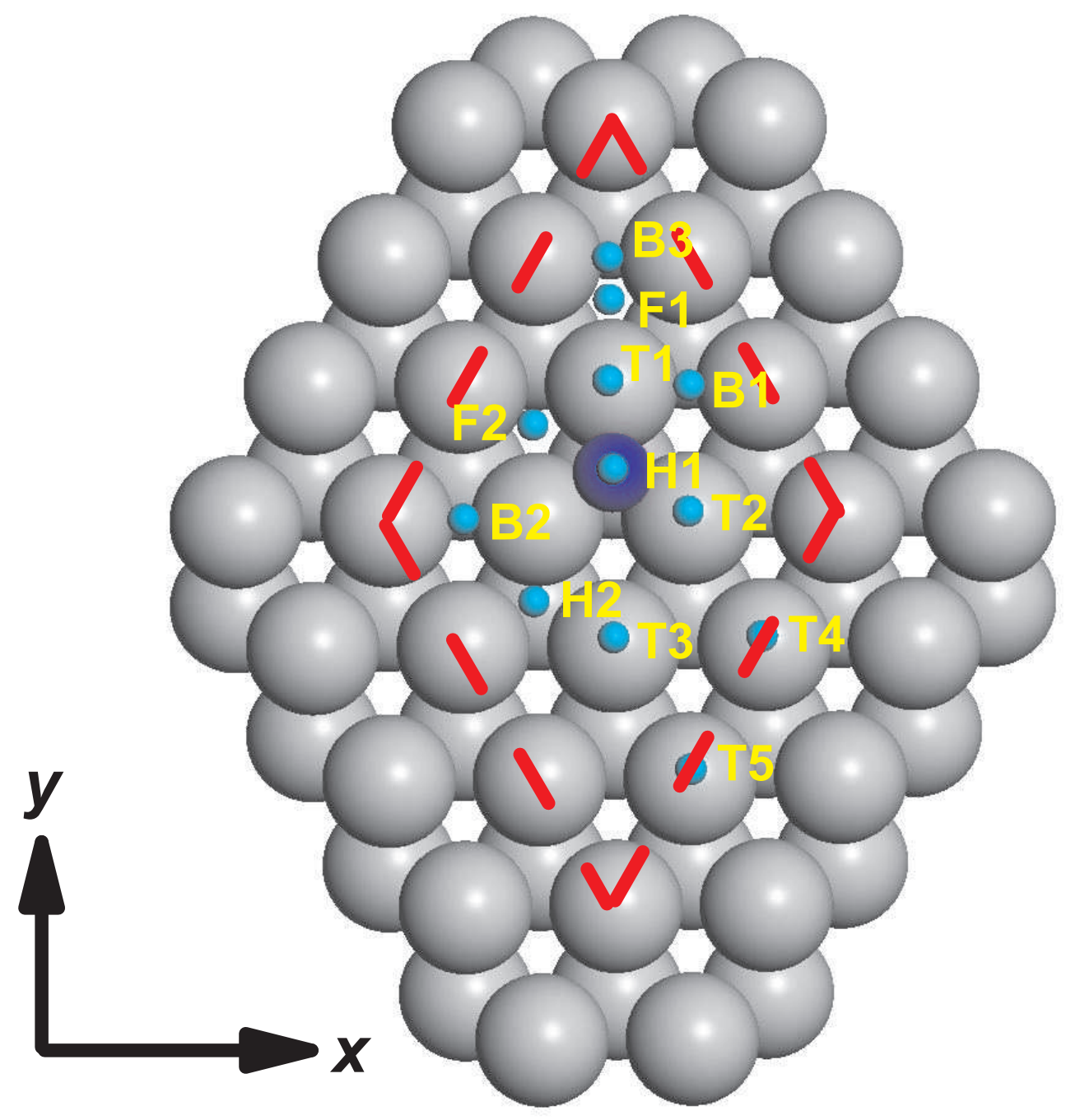

FIG. 7: (Color online). The schematic model for studying the adsorption of a $\mathrm{H}_{2}$ molecule on the hydrogen-preadsorbed $\mathrm{Be}(0001)$ surface. The blue and grey balls respectively represent $\mathrm{H}$ and Be atoms. And the light green balls are used to represent the high symmetry sites, that are denoted as $\mathrm{T} 1 \sim \mathrm{T} 5, \mathrm{~B} 1 \sim \mathrm{B} 3, \mathrm{~F} 1 \sim \mathrm{F} 2$ and $\mathrm{H} 1 \sim \mathrm{H} 2$.

than that on a clean $\operatorname{Be}(0001)$ surface. As shown in Figs. 8(c) and (d), the dissociation energy barrier for a $\mathrm{H}_{2}$ molecule along the B3-y and T3-x channels are respectively 0.85 and $0.50 \mathrm{eV}$, which are smaller than the dissociation energy barriers of a $\mathrm{H}_{2}$ molecule at the bridge and top site of a clean $\mathrm{Be}(0001)$ surface (i. e., 0.91 and $0.75 \mathrm{eV}$ respectively). The T3- $x$ channel is also the adsorption channel with the lowest energy barrier for dissociation of $\mathrm{H}_{2}$ on the preadsorbed $\mathrm{Be}(0001)$ surface. Considering that the dissociation of $\mathrm{H}_{2}$ molecules should not be affected by a preadsorbed $\mathrm{H}$ atom far away from them, and the dissociation 

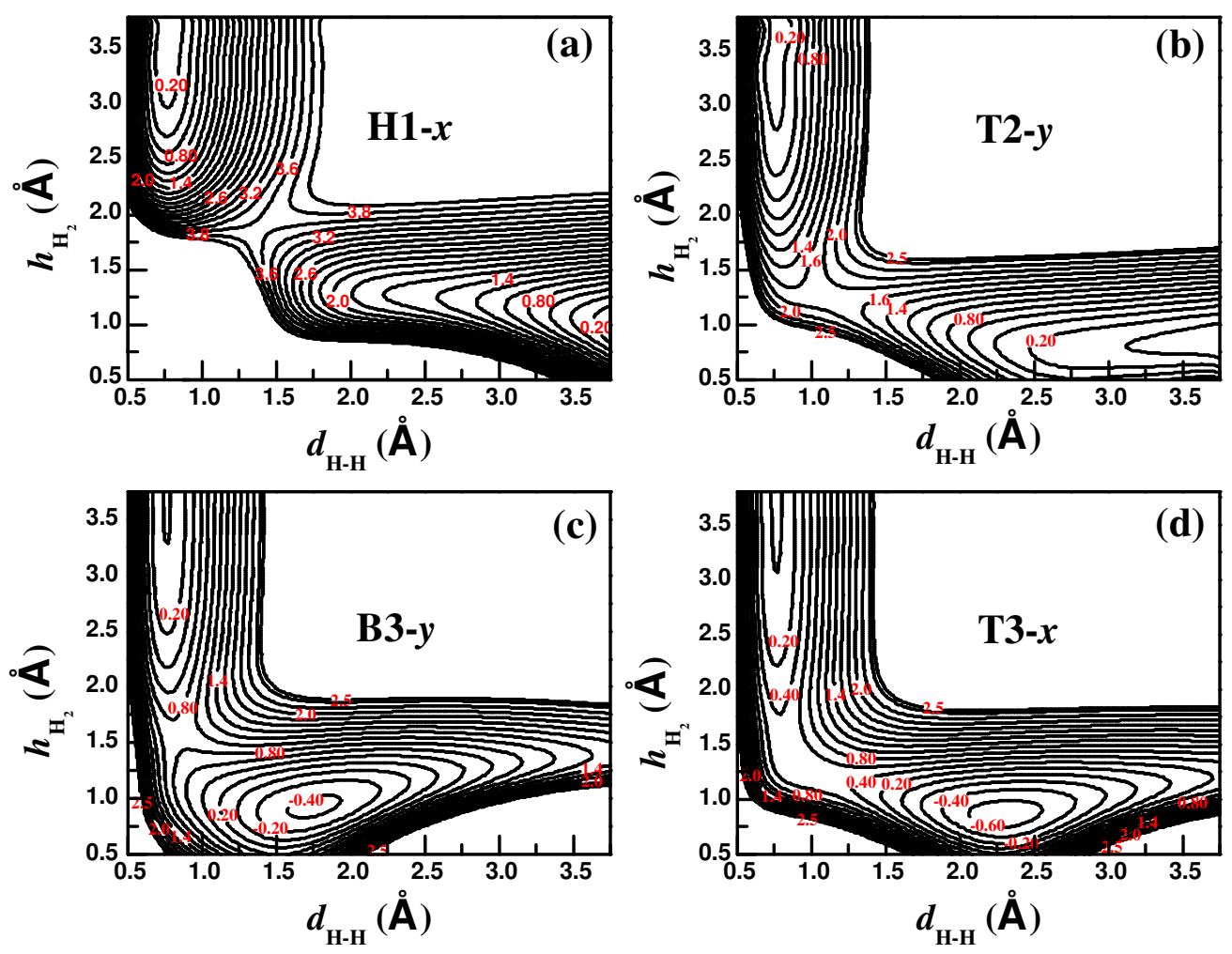

FIG. 8: (Color online). Contour plots of the two-dimensional cuts of the potential energy surfaces (PESs) for the $\mathrm{H}_{2} / \mathrm{Be}(0001)$ system as a function of the bond lengths $\left(d_{\mathrm{H}-\mathrm{H}}\right)$ and the heights $\left(h_{\mathrm{H}_{2}}\right)$, along the H1-x (a), T2-y (b), B3- $y$ and T3-x (d) channels.

energy barrier of a $\mathrm{H}_{2}$ molecule at the top site of a clean $\mathrm{Be}(0001)$ surface is $0.75 \mathrm{eV}$, the dissociation energy barrier for a $\mathrm{H}_{2}$ molecule will go back to $0.75 \mathrm{eV}$ when it is very far away from the preadsorbed hydrogen atom and adsorb at surface top sites. Therefore, we can see that the horizontal distances from the preadsorbed hydrogen atoms $\left(d_{0}\right)$ have critical effects on the dissociation energy barrier of adsorbing $\mathrm{H}_{2}$ molecules. The dissociation energy barriers $(\Delta \mathrm{E})$ for an $\mathrm{H}_{2}$ molecule along all the considered adsorption channels on the hydrogen-preadsorbed Be(0001) surface are summarized in Table II, together with the bond length $\left(d_{\mathrm{H}-\mathrm{H}}(\mathrm{TS})\right)$ and molecular height of $\mathrm{H}_{2}\left(h_{\mathrm{H}_{2}}(\mathrm{TS})\right)$ at the corresponding transition states.

To illustrate more specifically the dependence of the dissociation energy barrier for $\mathrm{H}_{2}$ molecules on their horizontal distances from the preadsorbed hydrogen atom (i.e., $d_{0}$ ), we then summarize the dissociation energy barriers for $\mathrm{H}_{2}$ molecules along all the considered adsorption channels together and show them in Fig. 9. Figure 9(a) shows the dissociation 
TABLE II: The dissociation energy barriers for $\mathrm{H}_{2}$ along the high symmetry adsorption channels on the hydrogen-preadsorbed $\mathrm{Be}(0001)$ surface, geometries for the corresponding transition states and corresponding horizontal distances from the preadsorbed hydrogen atom.

\begin{tabular}{|c|c|c|c|c|}
\hline $\begin{array}{c}\text { adsorption } \\
\text { channel }\end{array}$ & $\Delta \mathrm{E}(\mathrm{eV})$ & $\mathrm{I}(\mathrm{TS})(\AA)$ & $h_{\mathrm{H}_{2}}(\mathrm{TS})(\AA)$ & $d_{0}(\AA)$ \\
\hline $\mathrm{T} 1-x$ & 1.54 & 1.14 & 1.36 & 1.31 \\
\hline $\mathrm{T} 2-x$ & 0.69 & 1.89 & 1.23 & 1.31 \\
\hline $\mathrm{T} 2-y$ & 1.70 & 1.15 & 1.24 & 1.31 \\
\hline Т $3-x$ & 0.50 & 0.95 & 1.32 & 2.62 \\
\hline $\mathrm{T} 3-y$ & 0.68 & 1.22 & 1.29 & 2.62 \\
\hline $\mathrm{T} 4-x$ & 0.58 & 0.85 & 1.56 & 3.46 \\
\hline $\mathrm{T} 4-y$ & 0.60 & 0.88 & 1.56 & 3.46 \\
\hline $\mathrm{T} 5-x$ & 0.65 & 1.16 & 1.30 & 4.72 \\
\hline $\mathrm{T} 5-y$ & 0.56 & 1.06 & 1.38 & 4.72 \\
\hline B1-y & 1.21 & 0.90 & 1.45 & 1.73 \\
\hline $\mathrm{B} 2-y$ & 0.94 & 0.85 & 1.56 & 2.36 \\
\hline B3-y & 0.85 & 0.85 & 1.57 & 3.27 \\
\hline $\mathrm{F} 1-x$ & 2.06 & 1.34 & 1.36 & 1.31 \\
\hline $\mathrm{F} 2-x$ & 1.01 & 0.92 & 1.52 & 2.62 \\
\hline $\mathrm{H} 1-x$ & 3.61 & 1.45 & 1.91 & 0.00 \\
\hline $\mathrm{H} 1-y$ & 3.58 & 1.43 & 1.91 & 0.00 \\
\hline $\mathrm{H} 2-x$ & 1.14 & 1.00 & 1.35 & 2.27 \\
\hline $\mathrm{H} 2-y$ & 1.28 & 1.01 & 1.36 & 2.27 \\
\hline
\end{tabular}

energy barriers along all the top- $x(\mathrm{~T} 1 \sim \mathrm{T} 5-x)$ and top- $y(\mathrm{~T} 1 \sim \mathrm{T} 5-y)$ channels, from which one can see clearly that the dissociation energy barrier has the largest values when the $\mathrm{H}_{2}$ molecules are directly on top of the preadsorbed hydrogen atom, and the smallest values when the $\mathrm{H}_{2}$ molecules are about $3.50 \AA$ far away from the preadsorbed hydrogen atom. When the $\mathrm{H}_{2}$ molecules are over $4.00 \AA$ far away from the preadsorbed hydrogen atom, the dissociation energy barriers for them become larger again and approach the values on the clean $\mathrm{Be}(0001)$ surfaces. In this case, the dependence of the dissociation energy barriers for 

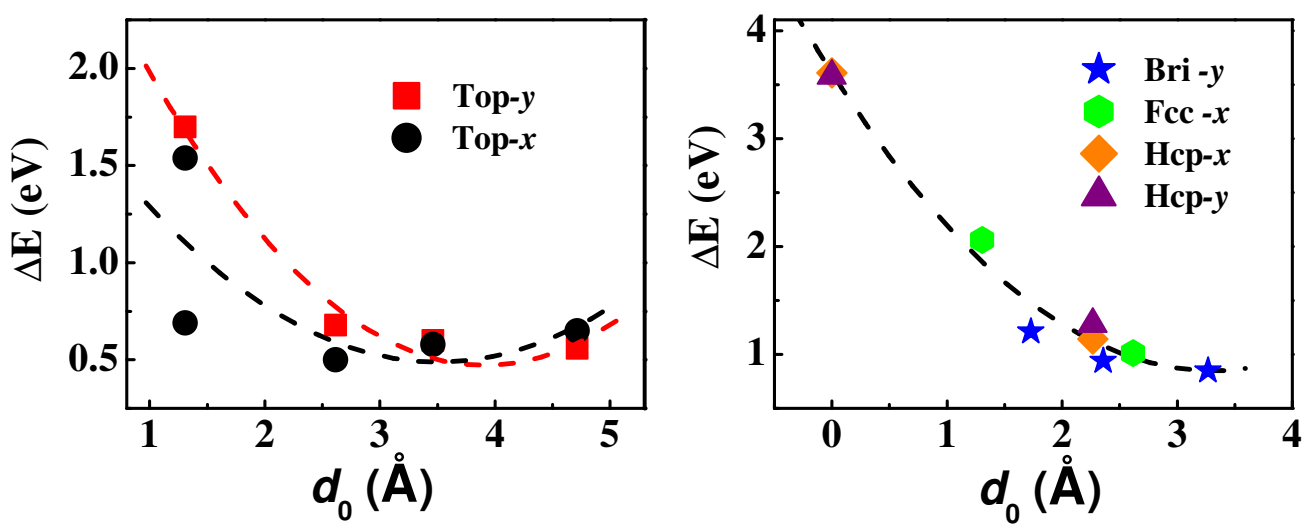

FIG. 9: (Color online). The dissociation energy barrier for $\mathrm{H}_{2}$ molecules along the T1 T5- $x, y$ channels (a) and other considered channels (b) as a function of their horizontal distances from the preadsorbed hydrogen atom.

$\mathrm{H}_{2}$ molecules on their horizontal distances from the preadsorbed hydrogen atom is clear. The dissociation energy barrier for $\mathrm{H}_{2}$ molecules along other adsorption channels are summarized in Fig. 9(b), from which the lowering down of the dissociation energy barrier with increasing $d_{0}$ is also very clear. However, the increasing of the dissociation energy barrier with very large $d_{0}$ is unseen because of the limitation of our cell size.

\section{CONCLUSIONS}

In conclusion, we have systematically investigated the dissociation of $\mathrm{H}_{2}$ molecules on the clean and hydrogen-preadsorbed Be(0001) surfaces by using the DFT methods. And our paper perfectly describes the dissociation behaviors for $\mathrm{H}_{2}$ molecules on the $\mathrm{Be}(0001)$ surface.

From the calculated 2D PES cuts for $\mathrm{H}_{2}$ molecules on the clean $\mathrm{Be}(0001)$ surface, we have found that surface top- $x, y$ channels are the most energetically favorable dissociation channels, and the lowest dissociation energy barrier is $0.75 \mathrm{eV}$ for the adsorbing $\mathrm{H}_{2}$ molecules. Besides, the dissociation of $\mathrm{H}_{2}$ along the bri- $y$ channel is also very probable to happen with the energy barrier of $0.91 \mathrm{eV}$. During our calculations, we have also found that the dissociation energy barrier for $\mathrm{H}_{2}$ at the hcp hollow sites is always smaller than at the fcc hollow sites on the clean Be(0001) surface.

For the dissociated hydrogen atoms, we have revealed that the diffusion energy barrier 
is as small as $0.35 \mathrm{eV}$, while the penetration energy barrier is as large as $1.25 \mathrm{eV}$, so the dissociated $\mathrm{H}$ atoms can easily diffuse around on the Be(0001) surface without penetrating into the subsurface sites. We have also revealed that the adsorption structure with uniform distributions of dissociated hydrogen atoms always has a lower energy.

Based on these studies, we have then further calculated the 2D PES cuts for $\mathrm{H}_{2}$ molecules on the hydrogen-preadsorbed Be(0001) surface, which shows that the dissociation energy barriers for $\mathrm{H}_{2}$ molecules depend critically on their horizontal distances from the preadsorbed hydrogen atom. The dissociation of $\mathrm{H}_{2}$ molecules directly on the preadsorbed hydrogen atom is very hard, but the lowest dissociation energy barrier for $\mathrm{H}_{2}$ on the hydrogen-preadsorbed Be surface is $0.25 \mathrm{eV}$ smaller than that on a clean Be surface.

\section{Acknowledgments}

This work was supported by the NSFC under Grant No. 10604010, No. 60776063, No. 50471070, No. 50644041, and by a fund from CAEP.

1 Hydorgen storage materials, edited by Z. L. Hu (Chemical Inustry Press, Beijing, 2002).

2 G. Lu and E. Kaxiras, Phys. Rev. Lett. 94, 155501 (2005).

3 The Chemical Physics of Solid Surfaces and Heterogeneous Catalysis, edited by D. A. King and D. P. Woodruff (Elsevier, Amsterdam, 1988).

4 K. Gustafsson and S. Andersson, Phys. Rev. Lett. 97, 076101 (2006).

5 T. Vegge, Phys. Rev. B 70, 035412 (2004).

6 M. Johansson, C. W. Ostenfeld and I. Chorkendorff, Phys. Rev. B 74, 193408 (2006).

7 G. X. Wu, J. Y. Zhang, Y. Q. Wu, Q. Li, G. Z. Zhou and X. H. Bao, Acta Phys. Chim. Sin. 24, 55 (2008).

8 E. V. Chulkov, V. M. Silkin, and E. N. Shirykalov, Surf. Sci. 188, 278 (1987).

9 A. Allouche, Phys. Rev. B 78, 085429 (2008).

10 R. A. Causey, J. Nucl. Mater. 300, 91 (2002).

11 F. S. Argentina, G. R. Longhurst, V. Shestakov, and H. Kawamura, J. Nucl. Mater. 283-287, 43 (2000). 
12 S. Zalkind, M. Polak, and N. Shamir, Surf. Sci. 513, 501 (2002).

13 S. Zalkind, M. Polak, and N. Shamir, Surf. Sci. 385, 318 (1997).

14 M. A. Gomez, L. R. Pratt, J. D. Kress, and D. Asthagiri, Surf. Sci. 601, 1608 (2007).

15 C. Linsmeier and J. Wanner, Surf. Sci. 454-456, 305 (2000).

16 S. Zalkind, M. Polak, and N. Shamir, Phys. Rev. B 71, 125413 (2005).

17 P. Zhang, B. Sun, and Y. Yang, Phys. Rev. B 79, 165416 (2009).

18 P. Feibelman, Phys. Rev. B 48, 11270 (1993).

19 R. Stumpf and P. J. Feibelman, Phys. Rev. B 51, 13748 (1995).

20 K. Pohl, E. W. Plummer, S. V. Hoffmann, and P. Hofmann, Phys. Rev. B 70, 235424 (2004).

21 G. Kresse and J. Furthmuller, Phys. Rev. B 54, 11169 (1996) and references therein.

22 J. P. Perdew and Y. Wang, Phys. Rev. B 45, 13244 (1992).

23 G. Kresse and D. Joubert, Phys. Rev. B 59, 1758 (1999).

24 H. J. Monkhorst and J, D. Pack, Phys. Rev. B 13, 5188 (1976).

25 M. Weinert and J. W. Davenport, Phys. Rev. B 45, 13709 (1992).

26 E. Wachowicz and A. Kiejna, J. Phys.: Condens. Matter 13, 10767 (2001).

27 K. P. Huber and G. Herzberg, Molecular Spectra and Molecular Structure IV: Constants of Diatomic Molecules (Van Nostrand Reinhold, New York, 1979).

28 M. Lazzeri and S. de Gironcoli, Phys. Rev. Lett. 81, 2096 (1998).

29 K. Pohl, J. H. Cho, K. Terakura, M. Scheffler, and E. W. Plummer, Phys. Rev. Lett. 80, 2853 (1998).

30 Y. Yang, G. Zhou, J. Wu, W. H. Duan, Q. K. Xue, B. L. Gu, P. Jiang, X. C. Ma, and S. B. Zhang, J. Chem. Phys. 128, 164705 (2008).

31 S. X. Wang, Y. Yang, B. Sun, R. W. Li, S. J. Liu, and P. Zhang (unpublished). 